\title{
Multimorbidity Patterns Among Hospitalized Geriatric Patients in Medicine Wards of a Tertiary Care Hospital in Dhaka City
}

\author{
Sumiya Bent Kalam, Sadia Islam², Mohammad Abdullah Az Zubayer Khan³, Tanjina Akhter \\ ${ }^{1}$ Assistant Professor, Department of Medicine, Delta Medical College Hospital, Dhaka, Bangladesh; ${ }^{2}$ Associate Professor, \\ Department of Medicine, Delta Medical College Hospital, Dhaka, Bangladesh; ${ }^{3}$ Assistant Professor, Department of \\ Haematology, National Institute of Laboratory Medicine and Referral Centre, Dhaka, Bangladesh; ${ }^{4}$ Consultant \\ (Medicine), Bangladesh Institute of Health Sciences General Hospital, Dhaka, Bangladesh
}

[Received on: 22 November 2020; Accepted on: 12 December 2020; Published: 1 January 2021]

\begin{abstract}
Background: Elderly people are posing a significant health burden in our country for their multi morbidity as economic growth has increased our life expectancy. Pattern of multi morbidity of this older people varies according to geography, ethnicity, culture and life style. Aim: The aim of this study was to identify the prevalence and pattern of multi morbidity of elderly patients admitted in a tertiary care hospital. Method: 50 random cases of elderly patients aged more than 60 years admitted in medicine and allied wards of Dhaka medical college hospital were observed at this cross-sectional study from January 2017 to June 2017(total 6 months period). Data were reviewed and analyzed using simple frequency and percentage. Protocol was reviewed by institutional ethical board (IRB) of Dhaka medical college hospital. Result: A total number of 50 elderly patients with age ranging from 60 to 95 years were observed with male and female ratio 1.3:1 having multi morbidity among $92.0 \%$ patients and female is more affected than male. Hypertension, ischemic heart disease, diabetes mellitus and stroke were found most common diseases as individual. Hypertension and diabetes mellitus was found as the most common multi morbidity pattern followed by hypertension and IHD, stroke \& diabetes mellitus. Conclusion: This study sheds light on priority needs of elderly patients in terms of medical facility in tertiary care hospital of Bangladesh. [Journal of National Institute of Neurosciences Bangladesh, January 2021;7(1): 56-59]
\end{abstract}

Keywords: : Multi-morbidity; geriatric patients; elderly patients

Correspondence: Dr. Sumiya Bent Kalam, Assistant Professor, Department of Medicine, Delta Medical College Hospital, Dhaka, Bangladesh; Cell no.: +8801915496525; Email: somumymen@gmail.com

Conflict of interest: None

Funding agency: Any of the author did not receive any external funding for this study.

Contribution to authors: Kalam SB was involved in the concept, study design and data collection. The rest of the authors were involved in data collection and partially writing and editing the manuscript.

How to cite this article: Kalam SB, Islam S, Khan MAAZ. Multi-morbidity Patterns Among Hospitalized Geriatric Patients in Medicine Wards of a Tertiary Care Hospital in Dhaka City. J Natl Inst Neurosci Bangladesh, 2021;7(1): 56-59

Copyright: (C2021. Kalam et al. Published by Journal of National Institute of Neurosciences Bangladesh. This article is published under the Creative Commons CC BY-NC License (https://creativecommons.org/licenses/by-nc/4.0/). This license permits use, distribution and reproduction in any medium, provided the original work is properly cited, and is not used for commercial purposes.

\section{Introduction}

Co-occurrence of two or more chronic diseases in a same individual is called multimorbidity. People with one chronic disease tends to have other one which is specially occur in elderly people. So the fact that more people are getting older, their disease pattern is changed from single comorbidity to multimorbidity ${ }^{1,2}$. By 2020 there will one billion elderly people throughout the world aged 60 years or more. Among them $70 \%$ will be from low income contries ${ }^{3}$. In Bangladesh the number of elderly people are projected to be from 7.8 million in 2001 to 16.2 million by 2025 as there is a significant improvement in mortality rate causing increased life expectancy ${ }^{4}$. Current data shows that life expectancy in Bangladesh is 71.8 years that would be higher in coming days making senior population a significant bulk of population. The elderly population is a high risk and vulnerable group in terms of health issues as they are more prone to suffer multimorbidity which consumes higher health care facility and health expenditure. 
Therefore the evaluation of present status of multimorbidity of our elderly population and their pattern is much warranted in current context.

Research on the multimorbidity pattern of geriatric people is very insufficient in south Asia including Bangladesh ${ }^{5}$. However, older citizen are important part of our country who need special care and attention. Most of them are not productive. Moreover if they suffer from multimorbidity it will put farther pressure on our poor economy and hamper our social atmosphere. For the last couple of years we are focusing on primary health care mainly maternal and child health. But geriatric health care facilities are being neglected. Lack of data delineating current health status of our elderly people and their impact on our economy is the key factor behind this. S this study will put some light on this aspect and help our policy makers to give emphasis on preparing intervention strategies for our geriatric population.

This study was undertaken to evaluate the scale of multimorbidity among hospitalized geriatric patients, to find out most common patterns of multimorbidity among hospitalized geriatric patients, and to see whether there is any significant relationship of multimorbidity with sex patterns.

\section{Methodology}

This was a small cross sectional study conducted in department of medicine of Dhaka medical college hospital from January 2017 to June 2017. Fifty elderly patients admitted in medicine indoor aged $\geq 60$ years from both sexes were included in this study by purposive sampling. Data was collected from the patient or from the attendance in case of inability of the patient to communicate. During data collection semi-structured questionnaire designed by the researcher containing relevant variables was used. All the patient was examined in accordance with history to get idea about the system of body involved, necessary findings were correlated with the information obtained and elaborated in admission ticket by bed-doctor.

\section{Results}

Data from fifty randomly selected patients with age ranging from 60 to 95 years were taken. Among them male was $28(56 \%)$ and female was $22(44 \%)$. Average age of male and female were 72 years and 68 years receptively. Highest age of male was 95 years and female was 80 years. Among 50 patients $46(92 \%)$ of patients had multimorbidity ( $\geq 2$ Diseases). Out of 28 male 24 had multimorbidity whereas all of the $(22$ in number) female had multimorbidity. One female patient had highest 5 systems involved. 4 systems were involved in 3 male patients and 6 female patients. Hypertension was found in 35 patients $(70 \%)$ which was most common health issue, among them $21(60 \%)$ patients were male and 14(40\%) were female. Diabetes mellitus was found in $24(48 \%)$ patients with equal male female ratio and diabetes was second most common disease after hypertension. Stroke was third most commonly involved with multisystem disorder $(n=23)$ involving $46 \%$ patients with female predominating, $70 \%$ vs $30 \%$. Ischemic heart disease was 4th common disease involving $21(42 \%)$ patients (male $62 \%$ and female $38 \%$ ) followed by arthritis $32 \%$ $(n=16)$, COPD 22\% $(n=11)$, CKD 20\%( $n=10)$ (Table 1).

Table 1: Distribution of Specific Disease with Sex $(n=50)$

\begin{tabular}{lccc}
\hline Disease & Male & Female & Total \\
\hline Hypertension & $21(\%)$ & $14(\%)$ & $35(70 \%)$ \\
Ischemic heart disease & $13(62 \%)$ & $8(38 \%)$ & $21(42 \%)$ \\
Stroke & $9(40 \%)$ & $14(60 \%)$ & $23(46 \%)$ \\
Dementia & $1(33 \%)$ & $2(67 \%)$ & $3(6 \%)$ \\
Arthritis & $7(44 \%)$ & $9(66 \%)$ & $16(32 \%)$ \\
Osteoporosis & $2(67 \%)$ & $1(33 \%)$ & $3(6 \%)$ \\
Diabetes mellitus & $12(50 \%)$ & $12(50 \%)$ & $24(48 \%)$ \\
COPD & $7(100 \%)$ & $0(0 \%)$ & $7(14 \%)$ \\
CKD & $4(50 \%)$ & $4(50 \%)$ & $8(16 \%)$ \\
Cancer & $2(67 \%)$ & $1(33 \%)$ & $3(6 \%)$ \\
Valvular Heart Disease & $0(0 \%)$ & $2(100 \%)$ & $2(4 \%)$ \\
\hline
\end{tabular}

Hypertension was found to be common in older patient group ( $\geq 70$ years) than less older age group (60-69 years) that is $67 \%$ vs $43 \%$. On the other hand, ischemic heart disease and stroke were found more common in less old $60-69$ years age group that is $52 \%$ vs $48 \%$ and $52 \%$ vs $48 \%$ consecutively. Arthritis and osteoporosis were also found more common in older age group 44\% vs $56 \%$ and $33 \%$ vs $67 \%$ consecutively. But diabetes mellitus was more common in less old age group 58\% vs $48 \%$ cases (Table 2 ).

Therefore the most common multimorbidity pattern was hypertension and diabetes seconded by hypertension and ischemic heart disease. 3rd most common pattern was stroke and diabetes followed by hypertension and arthritis. There were also other patterns of multimorbidity like hypertension, ischemic heart disease and CKD; hypertension, diabetes and CKD. Bronchial asthma, cataract, cancer, dementia, major depressive disorder was found sporadically along with other disease like hypertension, ischemic heart disease and diabetes mellitus (Table 3 ). 
Table 2: Distribution of Specific Disease and Age Group of Elderly $(\mathrm{n}=50)$

\begin{tabular}{lccc}
\hline Disease & Early Elderly & Late Elderly & Total \\
\hline Hypertension & $15(43 \%)$ & $20(57 \%)$ & $35(70 \%)$ \\
Ishchemic Heart Disease & $11(52 \%)$ & $10(48 \%)$ & $21(42 \%)$ \\
Stroke & $12(52 \%)$ & $11(48 \%)$ & $23(46 \%)$ \\
Dementia & $1(33 \%)$ & $2(67 \%)$ & $3(6 \%)$ \\
Arthritis & $7(43 \%)$ & $9(57 \%)$ & $16(32 \%)$ \\
Osteoporosis & $1(33 \%)$ & $2(67 \%)$ & $3(6 \%)$ \\
Diabetes Mellitus & $14(58 \%)$ & $10(42 \%)$ & $24(48 \%)$ \\
COPD & $7(100 \%)$ & $0(0 \%)$ & $7(14 \%)$ \\
Chronic Kidney Disease & $6(75 \%)$ & $2(25 \%)$ & $8(16 \%)$ \\
Cancer & $3(100 \%)$ & $0(0 \%)$ & $3(6 \%)$ \\
Valvular Heart Disease & $1(50 \%)$ & $1(50 \%)$ & $2(4 \%)$ \\
\hline
\end{tabular}

Early Elderly $=60-69$ years; Late Elderly $=\geq 70$ years

Table 3: Cross table delineating sex and system involved $(\mathrm{n}=50)$

\begin{tabular}{lccc}
\hline System Involved & Male & Female & Total \\
\hline 1 & $5(100 \%)$ & $0(0 \%)$ & 5 \\
2 & $10(59 \%)$ & $7(41 \%)$ & 17 \\
3 & $10(56 \%)$ & $8(44 \%)$ & 18 \\
4 & $3(33 \%)$ & $6(69 \%)$ & 9 \\
5 & $0(0 \%)$ & $1(100 \%)$ & 1 \\
\hline
\end{tabular}

\section{Discussion}

In this study it has been found that $92.0 \%$ of patients had multimorbidity. Among them all the female patients had multimorbidity. The statistics was higher from other studies like Khanam et $\mathrm{al}^{4}(53.8 \%)$ and Nwani et $\mathrm{al}^{6}$ $(49 \%)$ as these studies were conducted in a community level. Multimorbidity was found in almost all patients as this study was conducted in a tertiary care hospital which is in many cases probably the last destination of critically morbid patients in Bangladesh. Khanam ${ }^{4}$ also showed that prevalence of multimorbidity was significantly higher among woman.

Marengoni ${ }^{7}$ showed that hypertension, dementia, and heart failure were the most common disorders, with a prevalence of $38.0 \%, 21.0 \%$ and $18.0 \%$ respectively, whereas all other diseases were less frequent. The prevalence of hypertension is also higher in the late elderly age group, which is consistent with the findings showing that increasing age is a risk factor for hypertension ${ }^{8}$. Nimako ${ }^{9}$ also showed that the most common combination of conditions was hypertension \& diabetes mellitus (36.6\%), hypertension \& musculoskeletal conditions (19.9\%) and hypertension \& other cardiovascular conditions $(11.4 \%)$. Hossain et al10 showed that the combination of hypertension with cardiac diseases, hypercholesterolaemia with gouty arthritis were the most commonly occurring disease pairs in both sexes. Joshi et $\mathrm{a}^{11}$ also found that hypertension is one of the most prevalent part of multimorbidity among older followed by chronic obstructive airway disease, cataract and osteoarthritis. $\mathrm{He}$ also showed morbidities like asthma, COPD, hypertension, osteoarthritis, gastrointestinal disorders, anaemia, eye and neurological problems were significantly associated with disability and distress.

Higher number of morbidities was associated with greater disability and distress. About half of the patient in our study was suffering from DM with equal distribution of sex. It was second most common disease that we found in elderly patients. DM is as a part of metabolic syndrome is frequently found clustering with hypertension in many studies ${ }^{12}$. Stroke ranks third among causes of death in Bangladesh ${ }^{13}$. Mortality due to stroke has increased from $6.0 \%$ to around $9.0 \%$ from 2006 to $2011^{14}$. In Bangladesh people aged more than 40 years have a stroke prevalence of $0.3 \%$ and its prevalence increased to $1.0 \%$ in individuals aged 70 years or more ${ }^{15}$. Hospital-based studies conducted in past decades has indicated that hypertension and ishchemic heart disease is the main cause of ishchemic and haemorrhagic stroke in Bangladesh. So cardiovascular pattern comprising HTN and IHD, HTN and stroke, HTN, IHD and DM is the most common form of multimorbidity found in this study which is also revealed in other studies ${ }^{16-17}$.

\section{Conclusion}

This study gave interference about the depth of multimorbidity in elderly population of Bangladesh. Male and female were almost equally affected while combination of hypertension, DM and IHD were found to be most common multimorbidity patterns. Bangladesh is a developing country going through a transitional period where ageing population is gradually increasing due to increased life expectancy. They are more likely to be affected by multimorbidity. Therefore, vivid and realistic knowledge based on authentic data is crucial for comprehensive management of elderly patients.

\section{References}

1. Fortin M, Bravo G, Hudon C, et al. Prevalence of multimorbidity among adults seen in family practice. Ann Fam Med 2005;3:223-8

2. Wolff JL, Starfield B, Anderson G. Prevalence, expenditures, and complications of multiple chronic conditions in elderly. Arch Intern Med. 2002;162:2269-76 
3. Bloom BE, Canning D, Fink G. Implication of population ageing for economic growth. Oxf Rev Econ Policy 2010;26(4):583-612

4. Khanam MA, Streatfield PE, Kabir ZN, et al. Prevalence and pattern of multimorbidity among elderly people in rural Bangladesh: A Cross-Sectional Study. J Health Popul Nutr. 2011;29:406-14

5. Pati S, Swain S, Hussain MA, et al. Prevalence and Outcomes of Multimorbidity in South Asia: a systematic review. BMJ Open 2015;5(10)

6. Nwani PO, Isah AO. Chronic disease and multimorbidity among elderly patients admitted in the medical wards and multimorbidity among elderly patients admitted in the medical wards of Nigerian tertiary hospital. Journal of clinical Gerontology \& Geriatrics 2016;7(3):83-86

7. Marengoni A, Winblad B, Karp A, Fratiglioni L. Prevalence of chronic diseases and multimorbidity among the elderly population in Sweden. Am J Public Health 2008;98:1198-1200

8. William B Applegate. Hypertension. In: Beers MH, Berknow R, editors. The Merck manual of geriatrics. Pennsylvania: Merck \& Co. Inc., 2000:833

9. Nimako BA, Baiden F, Sackey SO, Binka F. Multimorbidity of chronic diseases among adult patients presenting to a inner-city clinic in Ghana. Glob Health 2013;9:61.

10. Hussain MA, Huxley RR, Mamun AA, Multimorbidity prevalence and pattern in Indonesian adults: an exploratory study using national survey data. BMJ Open 2015;5(12):9810

11. Joshi K, Kumar R, Kumar R, Avasthi A, Morbidity profile and its relationship with disability and psychological distress among elderly people in northern India. Int J Epidemoil 2003;32:978-87

12. Schafer I, Von Leitner EC, Schon G et al. Multimorbidity patterns in the elderly: a new approach of disease clustering identifies complex interrelations between chronic conditions. PLoS One 2010;5(12):15941

13. Islam MN, Moniruzzaman M, Khalil MI, Basri R, Alam MK, Loo KW et al. Burden of stroke in Bangladesh. International Journal of stroke.2013;8(3):211-213

14. Mohammad QD, Habib M, Hoque A, Hossain, et al. Prevalence of stroke above forty years. Mymensingh Medical Journal. 2011;20(4):640-644

15. Formiga F, Ferrer A, Sanz H, Marengoni A, et al. Patterns of comorbidity and multimorbidity in the oldest old: the orthodox study.Europian Journal of Internal Medicine.2013;24:40-4

16. Marengogni A, Rizzuto D, Wang HX, et al. Patterns of chronic multimorbidity in the elderly population. J Am Geriatr Soc.2009;57:225-30

17. John R, Kerby DS, Hennessy CH. Patterns and impact of comorbidity and multimorbidity among community-resident American Indian elders. Gerontologist 2003;43:649-60 
